\title{
The Under-Utilization of the Head Impulse Test in the Emergency Department
}

\author{
Timothy McDowell, Fraser Moore
}

\begin{abstract}
Background: The head impulse test (HIT) is an evidenced based clinical tool to differentiate between peripheral and central causes of vertigo. Our objective was to determine the rate of utilization of the HIT in the emergency room (ER). Methods: A retrospective chart review of patients presenting to the ER over one year who received a final diagnosis of dizziness or vertigo. Details of clinical examinations, investigations, and diagnosis were recorded. Patients were grouped into episodic, acute constant, and chronic vertigo groups. Results: HIT was performed in only 31 of $642(5 \%)$ patients with vertigo. In the acute constant group it was negative in 6 of 6 patients ultimately diagnosed with stroke and positive in 6 of 13 cases of peripheral vertigo. Discussion: Despite good published evidence regarding its use the HIT is under-utilized in the ER. Physicians need to be aware of the HIT and newer video HITs and make use of them in practice.
\end{abstract}

RÉSUMÉ: La sous-utilisation du test d'impulsion rotatoire de la tête au service des urgences. Contexte: Le test d'impulsion rotatoire de la tête (TIRT) est un outil clinique fondé sur des données probantes pour différencier les causes périphériques des causes centrales du vertige. Notre objectif était de déterminer le taux d'utilisation du TIRT au service des urgences (SU). Méthode: Nous avons effectué une revue rétrospective de dossiers de patients ayant consulté au SU au cours d'une période de un an et chez qui le diagnostic final était des étourdissements ou du vertige. Nous avons recueilli les détails de l'examen clinique, des explorations paracliniques ainsi que le diagnostic. Nous avons regroupé les patients selon que la sensation de vertige était épisodique, aiguë et constante ou chronique. Résultats: Le TIRT a été effectué chez seulement 31 des 642 patients (5\%) présentant un vertige. Dans le groupe où le vertige était aigu et constant, le test était négatif chez 6 patients parmi les 6 patients chez qui un diagnostic d'accident vasculaire cérébral a finalement été posé et positif chez 6 patients parmi les 13 patients atteints de vertige d'origine périphérique. Discussion: Malgré qu'il existe des données publiées de bonne qualité concernant l'utilisation du TIRT, il est sous-utilisé au SU. Les médecins doivent connaître le TIRT et le TIRT avec enregistrement vidéo et utiliser ces examens dans leur pratique.

Keywords: neurological examination, stroke, vestibular

doi:10.1017/cjn.2015.330

Can J Neurol Sci. 2016; 43: 398-401

Dizziness is a common presenting complaint to the emergency room (ER). ${ }^{1}$ Vertigo is one form of dizziness and can be divided, based on the timing of the symptoms, into episodic, acute constant, and chronic forms. Diagnosis and management of these patients in the ER is often inadequate. ${ }^{2}$ For example, specific guidelines exist for the use of the Dix-Hallpike maneuver followed by a particle repositioning (Epley) maneuver in the diagnosis and treatment of benign paroxysmal positional vertigo (BPPV). ${ }^{3}$ A recent population-based study has shown that these manoeuvres are underutilized in the ER. ${ }^{4}$

The acute vestibular syndrome (AVS) is a form of acute, constant vertigo that can be benign or due to life threatening causes such as posterior fossa stroke. ${ }^{5}$ The head impulse test (HIT) is an evidenced based clinical tool that is used to differentiate between patients with benign and serious causes of the AVS. ${ }^{6-8}$ A positive test occurs when a subject makes a voluntary corrective saccade to maintain fixation after passive head turning performed by the examiner. Guidelines have been published regarding its use. ${ }^{9}$ To our knowledge, there have been no studies investigating the utilization of the HIT in the ER.

We retrospectively analyzed the charts of patients who received a final diagnosis of dizziness or vertigo. Our goal was to identify the utilization rate of the head-impulse test. Secondary goals were to assess the use of the Dix-Hallpike Maneuver, the use of imaging, and stroke risk in these patients.

\section{Methods}

\section{Study Population}

We analyzed ER visits between January $1^{\text {st }} 2011$ and December $31^{\text {st }} 2011$ at the Jewish General Hospital, in Montreal QC Canada. The Jewish General Hospital is a 637 bed tertiary-care centre in Montreal affiliated with McGill University. There are approximately 67,000 ER visits annually. There are no neuro-otologists affiliated with the hospital, nor is there a referral bias for stroke, dizziness or vertigo patients that we are aware of. All ER visits with a final diagnosis of dizziness or vertigo based upon the International Classification of Diseases, ninth edition (ICD-9) coding system (780.4 and 386), were identified and included. Patients were excluded if a non-neurovestibular diagnosis (for

\footnotetext{
From McGill University, Department of Neurology, Montreal, Quebec, Canada Received April 8, 2015. Final Revisions Submitted August 6, 2015. Date of Acceptance August 6, 2015.

Correspondence to: Fraser Moore, Department of Neurology, McGill University, 3755 CoteSte-Catherine E-005, Montreal, QC, Canada, H3T 1E2. Email: fraser.moore@mcgill.ca
} 
example, syncope) was established immediately after the initial ER assessment. The study was approved by the ethics committee of the Jewish General Hospital.

\section{Data Collection}

A Microsoft Access ${ }^{\circledR}$ database of ER visits for dizziness or vertigo was created. Data abstraction was performed by one of the authors (TM) who was not blinded. The patients were grouped based on symptom duration into episodic, acute constant, or chronic categories based upon the initial treating physician's assessment in the ER. "Episodic" was defined as recurrent episodes of vertigo each lasting less than one hour. "Acute constant" was arbitrarily defined as a sudden onset of constant vertigo lasting for less than two weeks prior to presentation to the ER. "Chronic" was defined as constant vertigo lasting for more than two weeks.

The following information was abstracted independently from the ER physician and neurologist notes: presence of documented vascular risk factors, results of general neurology examination, eye movement examination, head impulse test, Dix-Hallpike maneuver, and tests of skew. The use and results of computed tomography $(\mathrm{CT})$ or magnetic resonance (MR) imaging was recorded. Imaging results were classified as "normal", "abnormal but no change from previous", "incidental findings", "findings suggestive of posterior circulation ischemia or hemorrhage", or "other relevant". The final diagnosis was abstracted from the neurologist's note, or the ER note when no neurological consultation was performed. If repeat ER visits occurred related to the same symptoms then the final diagnosis was used. Vestibular neuritis, vestibular neuropathy, labyrinthitis, and viral vestibulopathy were all grouped together as vestibular neuritis. BPPV, benign positional vertigo, and positional vertigo were grouped together as BPPV.

\section{ResUlts}

We identified 1196 patients with a final diagnosis of dizziness or vertigo (Figure). From the latter, 10 charts were excluded due to illegibility of the notes. Of the remaining 1186, 544 did not have a vestibular diagnosis. Six hundred and forty-two patients with vertigo remained. This cohort was $60.3 \%$ female, with an average age of 63. Two hundred and forty-five patients were classified as episodic vertigo, 260 as acute constant vertigo, and 87 as chronic. For 50 patients, information regarding timing was unavailable.

\section{Utilization of head impulse test (HIT)}

The HIT was performed a total of 34 times in 31 of the 642 patients $(5 \%)$ with vertigo. It was performed 24 times by neurologists (out of a total of 105 neurology consults done on the 642 total patients) and 10 times by ER physicians. It was performed in 8 of 245 (3\%) episodic vertigo patients, in 19 of 260 (7\%) acute constant vertigo patients, and in 4 of 87 (5\%) chronic vertigo patients. The Table provides a breakdown of positive and negative HIT results by group and provides the final diagnoses.

The HIT is most appropriately used in the setting of acute constant vertigo. ${ }^{6,8}$ For these patients, the HIT was negative in all 6 patients in whom it was performed who were diagnosed with stroke. It was positive in 6 of the 13 remaining cases in which it was performed. All 13 of these non-stroke, acute constant vertigo cases received a final diagnosis of peripheral vertigo (Table). The HIT results in stroke versus non-stroke acute constant vertigo cases did not reach statistical significance (Fisher exact test $\mathrm{p}=0.06$ ).

\section{Utilization of other bedside testing}

In the episodic vertigo cases, the Dix-Hallpike maneuver was performed in 77 (31.4\%) patients. The Epley maneuver was performed in $15.3 \%$ of patients diagnosed with BPPV.

\section{Utilization of Imaging}

A CT scan was performed in 76 of 245 (31.0\%) episodic vertigo patients. None were found to have significant lesions. Magnetic resonance imaging was performed in three of these patients and none were found to have significant lesions. In the acute constant subgroup, 132 of $260(50.8 \%)$ patients had CT scans with 5 (3.8\%) having significant lesions, all ischemic stroke. Twenty-five (9\%) patients had an MR imaging brain with $12(48.0 \%)$ showing significant lesions. Fifty three of 87 chronic vertigo patients had a CT, with the only abnormality being an incidental orbital lesion on one CT.

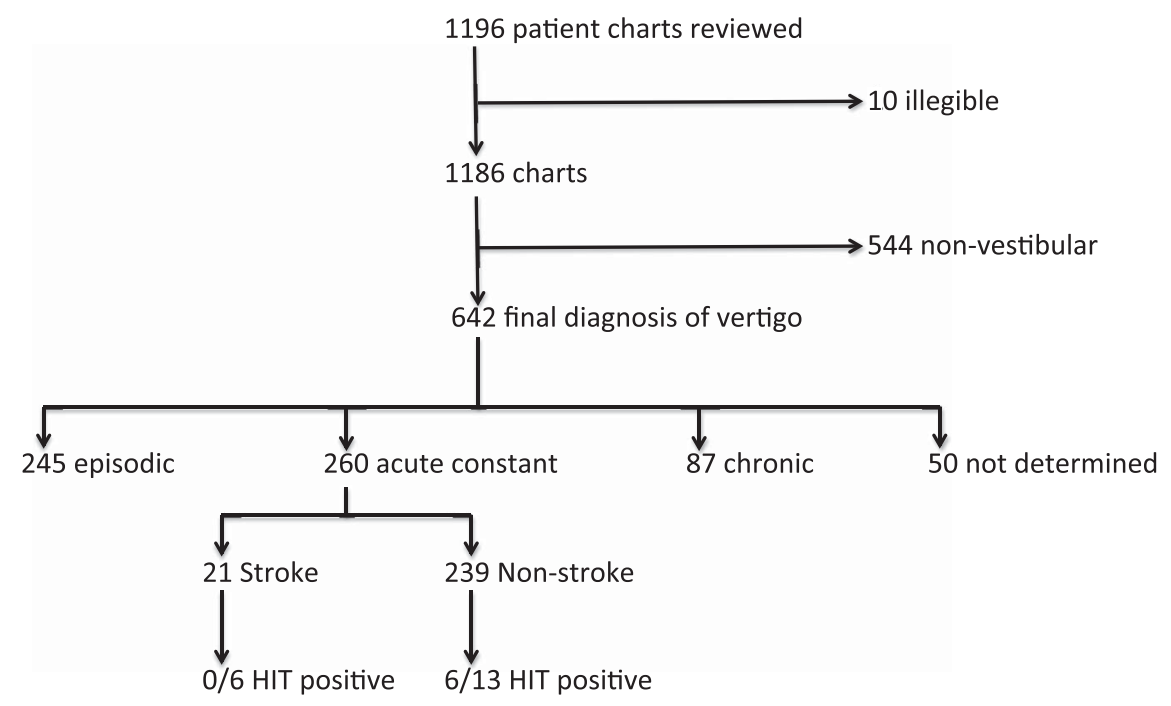

Figure: Results of chart review of all emergency room visits in 2011 with a final diagnosis of vertigo. 


\section{Table: Final diagnosis of 31 patients who had Head Impulse Testing (HIT) in the Emergency Room and received a discharge diagnosis of dizziness or vertigo. Patients are divided by subgroup of vertigo and result of HIT.}

\begin{tabular}{l|c|c}
\hline Vertigo & Positive HIT & Negative HIT \\
\hline Episodic & $\mathbf{2}$ Total & $\mathbf{6}$ Total \\
\hline & 1 BPPV & 3 BPPV \\
\hline & 1 Vestibular Neuritis & 1 Vestibular Neuritis \\
\hline & & 2 "Peripheral vertigo" \\
\hline Acute Constant & $\mathbf{6}$ Total & $\mathbf{1 3}$ Total \\
\hline & 5 Vestibular Neuritis & 6 Stroke \\
\hline & 1 "Peripheral vertigo" & 3 Vestibular Neuritis \\
\hline & & 2 "Peripheral vertigo" \\
\hline Chronic & & 2 No diagnosis \\
\hline & $\mathbf{1 ~ T o t a l ~}$ & $\mathbf{3}$ Total \\
\hline & 1 Vestibular Neuritis & 1 BPPV \\
\hline & & 1 "Peripheral vertigo" \\
\hline
\end{tabular}

HIT $=$ Head impulse test

$\mathrm{BPPV}=$ Benign paroxysmal positional vertigo

Performing a CT scan extended the average time in ER from 4:25 to 9:16 hours (excluding the admitted stroke patients).

\section{Risk of Stroke}

The overall risk of stroke was $3.3 \%$ (21/642), with all strokes occurring in the acute constant subgroup $(21 / 260,8.1 \%)$. As noted, the HIT was negative in all six stroke patients in whom it was performed. A CT scan was positive in 5 of the $21(2.4 \%)$ stroke patients. Magnetic resonance imaging was positive in 10 of the 15 stroke patients in whom it was performed.

Independent review of all 308 stroke admissions to the hospital in 2011 only identified a single additional patient who had presented with chronic vertigo but was not identified in our series of ER visits. (This case is not included among our 642 cases).

\section{DISCUSSION}

Careful attention to both the history and the use of validated bedside tests can lead to appropriate diagnosis and treatment of patients presenting to the ER with dizziness and vertigo. None of the patients with episodic or chronic vertigo in our study were diagnosed with stroke or other serious intracranial cause. Of the 21 patients presenting with acute constant vertigo who were diagnosed with stroke, the HIT was negative in each of the 6 patients in whom it was done while it was positive in 6 of 13 non-stroke cases. These findings agree with previous studies ${ }^{7,9,10}$ that patients presenting with acute, constant vertigo and a negative HIT have a much higher chance of stroke as a cause of their symptoms compared to other vertigo patients.

Unfortunately, this study clearly demonstrates that the head-impulse test (HIT) was under-utilized by both ER physicians and neurologists when evaluating patients presenting with acute constant vertigo in the emergency room. It was utilized in only
19 of 260 such cases. To our knowledge the utilization of the HIT has not been reported previously. We also found that the Dix-Hallpike maneuver was underutilized, being performed in only $31 \%$ of patients with episodic vertigo. This agrees with a previous US study in the United States (US), in which only $21.8 \%$ of patients diagnosed with BPPV underwent the Dix-Hallpike maneuver. ${ }^{4}$ There are probably multiple reasons for the underutilization of bedside tests such as the HIT. Physicians may be unaware of the test, lack confidence in performing it, or they may consider it to be too subjective and prefer to rely on their history. As the HIT is a simple and rapid test to administer it is unlikely that time was a factor.

The implication of more appropriate use of history and bedside testing are obvious. Computed tomography scanning was performed in 76 episodic vertigo and 53 chronic vertigo patients, with none showing a significant abnormality except for a single incidental orbital lesion. The low yield of CT scanning in this patient population agrees with previous studies. ${ }^{11,12}$ Avoiding imaging in these cases would have a direct cost savings and in our study would have reduced the average time spent in the ER by half. The utilization of CT head imaging was higher in our study (31\% and 50\% in the episodic and acute constant subgroups, respectively) compared to a US population study (25\%). ${ }^{1}$ This was unexpected as ER CT use in Canada is typically lower than in the US. ${ }^{13}$ One explanation may be that our cohort comprises patients with a final diagnosis of dizziness or vertigo rather than a presenting complaint of dizziness or vertigo.

This study has several limitations. It was retrospective and non-blinded. Our analysis was based on written documentation, although there is some published evidence that this correlates well with the medical encounter. ${ }^{14}$ The small number of patients who underwent HIT makes it difficult to draw definite conclusions about its usefulness, but this was not the primary objective of the study and has been previously reported ${ }^{7,9}$. We do not know how many physicians were aware of the HIT and chose not to use it, as opposed to being unaware of it. There is no definitive gold standard test for many of the final diagnoses made in dizzy patients and, to our knowledge, the majority of our subjects with a final diagnosis of peripheral vertigo did not have follow-up imaging (CT or MRI) in the days following their ER visit to assure that the diagnosis of peripheral disease was not incorrect.

Recent publications have described the use of the video head impulse test (vHIT). ${ }^{15-17}$ This uses a head-mounted camera to measure eye movements during the head turn. It has the advantage of being able to detect "covert saccades" that the examiner would miss by visual inspection alone. ${ }^{8}$ The vHIT can reliably distinguish vestibular neuritis and stroke and a clinical trial regarding its use is underway. ${ }^{18}$ We would certainly encourage the use of the vHIT where available. However, the results of our study with bedside (non-video) HIT are still relevant for two reasons. If physicians so dramatically under-utilize a simple bedside clinical test like the HIT it seems unlikely they would be more likely to utilize an updated version requiring digital equipment and analysis. More importantly, this technology is unlikely to be available in every ER. We suggest the following approach to patients presenting to the ER with an acute vestibular syndrome: A careful history regarding symptom timing and duration to identify those with acute, constant vertigo; a bedside HIT; and, where available, a video HIT. 


\section{DISCLOSURE INFORMATION}

Fraser Moore and Timothy McDowell do not have anything to disclose.

\section{REFERENCES}

1. Kerber KA, Meurer WJ, West BT, Fendrick AM. Dizziness presentations in U.S. emergency departments, 1995-2004. Acad Emerg Med. 2008;15:744-50.

2. Newman-Toker DE, Hsieh YH, Camargo CA, et al. Spectrum of dizziness visits to US emergency departments: cross-sectional analysis from a nationally representative sample. Mayo Clin Proc. 2008;83:765-75.

3. Bhattacharyya N, Baugh RF, Orvidas L, et al. Clinical practice guideline: benign paroxysmal positional vertigo. Otolaryngol Head Neck Surg. 2008;139(Suppl 4):S47-81.

4. Kerber KA, Burke JF, Skolarus LE, et al. Use of BPPV processes in emergency department dizziness presentations: a populationbased study. Otolaryngol Head Neck Surg. 2013;148:425-30.

5. Kattah JC, Talkad AV, Wang DZ, Hsieh YH, Newman-Toker DE. HINTS to diagnose stroke in the acute vestibular syndrome: threestep bedside oculomotor examination more sensitive than early MRI diffusion-weighted imaging. Stroke. 2009;40:3504-10.

6. Halmagyi GM, Curthoys IS. A clinical sign of canal paresis. Arch Neurol. 1988;45:737-9.

7. Newman-Toker DE, Kattah JC, Alvernia JE, Wang DZ. Normal head impulse test differentiates acute cerebellar strokes from vestibular neuritis. Neurology. 2008;70:2378-85.

8. Weber KP, Aw ST, Todd MJ, et al. Head impulse test in unilateral vestibular loss. Vestibulo-ocular reflex and catch-up saccades. Neurology. 2008;70:454-63.
9. Tarnutzer AA, Berkowitz AL, Robinson KA, et al. Does my dizzy patient have a stroke? A systematic review of bedside diagnosis in acute vestibular syndrome. CMAJ. 2011;183:E571-92.

10. Chen L, Lew W, Chambers BR, Dewey HM. Diagnostic accuracy of acute vestibular syndrome at the bedside in a stroke unit. J Neurol. 2011;258:855-61.

11. Kerber KA, Schweigler L, West BT, et al. Value of computed tomography scans in ED dizziness visits: analysis from a nationally representative sample. Am J Emerg Med. 2010;28:1030-6.

12. Lawhn-Heath C, Buckle C, Christoforidis G, Straus C. Utility of head CT in the evaluation of vertigo/dizziness in the emergency department. Emerg Radiol. 2013;20:45-9.

13. Berdahl CT, Vermeulen MJ, Larson DB, Schull MJ. Emergency Department Computed Tomography Utilization in the United States and Canada. Ann Emerg Med. 2013;62:486-94.

14. Stange KC, Zyzanski SJ, Smith TF, et al. How valid are medical records and patient questionnaires for physician profiling and health services research? A comparison with direct observation of patients visits. Med Care. 1998;36:851-67.

15. MacDougall HG, Weber KP, McGarvie LA, et al. The video head impulse test. Diagnostic accuracy in peripheral vestibulopathy. Neurology. 2009;73:1134-41.

16. MacDougall HG, McGarvie LA, Halmagyi GM, et al. The video head impulse test (vHIT) detects vertical semicircular anal dysfunction. PLoS One 2013;8:e61488.

17. Matino-Soler E, Esteller-More E, Martin-Sanchez JC, et al. Normative data on angular vestibulo-ocular responses in the yaw axis measured using the video head impulse test. Otol Neurotol. 2015;36:466-71.

18. Mantokoudis G, Saber Tehrani AS, Wozniak A, et al. VOR gain by head-impulse video-oculography differentiates acute vestibular neuritis from stroke. Otol Neurotol. 2015;36:457-65. 